Classification

Physics Abstracts

$68.45-61.40-61.30-76.00$

\title{
Surface induced disorder of nematic MBBA near silica with grafted poly(ethylene oxide)
}

\author{
H. Ben Ouada, H. Hommel, A. P. Legrand \\ Laboratoire de Physique Quantique, U.A. 421 C.N.R.S., \\ E.S.P.C.I., 10, rue Vauquelin, 75231 Paris Cedex 05, France \\ H. Balard and E. Papirer \\ Centre de Recherches sur la Physico-Chimie des surfaces solides, \\ L.P. 6601 C.N.R.S., 24, avenue du Président Kennedy, 68200 Mulhouse, France
}

(Reçu le 2 mai 1985, accepté sous forme définitive le 8 janvier 1986)

\begin{abstract}
Résumé. - L'ordre d'un cristal liquide nématique, le MBBA, au contact d'une surface solide de silice couverte de polymères greffés, du polyoxyde d'éthylène 2000 , a été étudié à l'aide des techniques suivantes : la résonance paramagnétique électronique, la résonance magnétique nucléaire et l'analyse calorimétrique différentielle. Les différents spectres, les températures de transitions et le degré d'ordre des molécules sont indiqués. Les résultats sont cohérents avec une image globale de l'interface consistant en une couche intermédiaire, formée par le polymère mélangé au cristal liquide désordonné. Le greffage de polymères améliore beaucoup l'orientation du cristal liquide nématique en volume et diminue l'influence de la surface.
\end{abstract}

\begin{abstract}
The order of a nematic liquid crystal, MBBA, in contact with a solid silica surface covered with grafted polymers poly(ethylene oxide) 2000 , has been investigated by the electron paramagnetic resonance, the nuclear magnetic resonance and the differential scanning calorimetry. The different spectra, the transitions temperatures and the degree of order of the molecules are given. The results are consistent with an overall picture of the interface consisting of an intermediate layer formed by polymer mixed with disordered liquid crystal. The grafting of polymers greatly improves the decoupling of the bulk nematic liquid crystal from the surface.
\end{abstract}

\section{Introduction}

It is now well established that solid surfaces can have an aligning effect for the molecules of liquid crystal in contact. Homeotropic (perpendicular to the surface), homogeneous or planar (parallel to the surface) and tilted orientations have been described [1]. The orientation depends strongly on the state of the solid surface. The latter can be modified by mechanical action (rubbing...) [2, 3], chemical treatments, film deposition [4] or by adsorption of surfactants on the surface [5-11]. Several methods have been proposed for measuring the anchoring energy [12-15]. Often the order parameter is larger at the surface than in the bulk [16-18]. Generally these surface effects are important for the liquid crystal device applications, and are studied on macroscopic surfaces (glass plates). A definite orientation over a large range is required for these applications.

Here our purpose is somewhat different. This paper describes the effect of macromolecules, namely poly(ethylene oxide), grafted i.e. chemically bonded, onto the solid surface, on the orientation properties of a liquid crystal. As we want to investigate only the local order, we have chosen microscopic silica surfaces. It has already been shown that macromolecules in solution in liquid crystal destroy the nematic order in their vicinity [19-21]. Kronberg et al. [19] have studied the system EBBA + poly(ethylene oxide). They have shown that it seems that the size rather than the nature of the segments is important in destroying the order in the nematic phase. It is therefore expected that grafted polymers will, on the contrary of the usual action, decouple the solid surface from the bulk liquid crystal [22].

Three different methods are used : the differential scanning calorimetry (DSC), the electronic paramagnetic resonance (EPR) of labels or probes, and the nuclear magnetic resonance (NMR) of the protons. The first method will give global informations whereas 
the latter yields local microscopic ones. For DSC there is no external field and the liquid crystal is only influenced by the presence of the modified silica surface. With EPR and NMR an external magnetic field of $3250 \mathrm{G}$ is applied, which tends to align the liquid crystal molecules in its own direction. The average alignment direction, which results from the combined action of the surface and the field, will not be the same at different points of the samples.

\section{Experimental.}

2.1 Materials. - The samples of grafted polymers were the same as used previously (23-25).

2.1.1 Silica. - The silica used was pyrogenic, non microporous, Aerosil 300 from Degussa. It consists of clusters of small spheres of about $10 \mathrm{~nm}$ diameter. The specific area measured by nitrogen adsorption was about $310 \mathrm{~m}^{2} / \mathrm{g}$.

2.1.2 Polymers. - The polymer was poly(ethylene oxide) (PEO) of molecular weight 2000 containing 45 repetition units. Its polydispersity was equal to 1.04 . The polymer was heat dried at $70^{\circ} \mathrm{C}$, under vacuum for about $3 \mathrm{~h}$.

2.1.3 Grafting reaction. - Before the grafting, the silica was dehydrated by heating at $300^{\circ} \mathrm{C}$ under vacuum for 3 hours. The grafting reaction was the direct esterification of the silanol groups by the hydroxyl end groups of the polymers. Practically, the silica was dispersed in an excess of PEO and the dispersion was carefully degassed and heated at $230^{\circ} \mathrm{C}$ for 16 hours under pure nitrogen to prevent oxidation. The products of the reaction were then dispersed in aceton, filtered and the filtrate washed three times and solvent extracted. The grafted silica was then heated at $60^{\circ} \mathrm{C}$ and dried under vacuum. The grafting ratio determined by pyrolysis weight loss at $700{ }^{\circ} \mathrm{C}$ was $0.19 \mathrm{molecule} / \mathrm{nm}^{2}$ i.e. the Gaussian coils are weakly interacting and form a relatively narrow layer at the interface.

2.1.4 Spin-labelling. - These grafted chains were then labelled at the free end by the nitroxide free radical 2-2-5-5 tetramethyl-3-pyrroline-1-oxyl-3-carboxylic acid from Eastman in its acid chloride form. In order to avoid physical interaction between neighbouring labels, about one percent of the chains were labelled. The same nitroxide radical was also used as spin-probe in pure MBBA (control). The chemical formula of a grafted, labelled polymer chain is given in figure 1.

2.1.5 Liquid crystal. - The liquid crystal was pmethoxybenzylidene-p-n-butylaniline (MBBA) from Aldrich, which was nematic between $21^{\circ} \mathrm{C}$ and $44^{\circ} \mathrm{C}$ and used as received. The MBBA is a solid below $21^{\circ} \mathrm{C}$ and an isotropic liquid above $44^{\circ} \mathrm{C}$. This liquid crystal, well known, was chosen because its nematic temperature range was sufficiently low and compatible with the stability of the grafting and of the

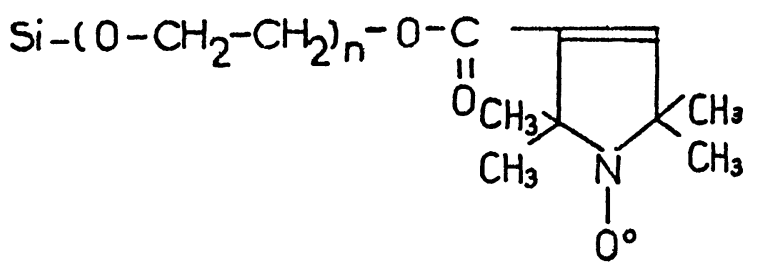

Fig. 1. - Chemical formula of a grafted, labelled, PEO chain.

label. It is known that this nematic liquid crystal is in average aligned along the direction of an applied external magnetic field of $3250 \mathrm{G}$ [26].

The chemical formula of the MBBA molecule is given in figure 2.

\section{Electronic paramagnetic resonance.}

3. 1 Samples and apparatus. - Two EPR samples were prepared in pyrex tubes of $4 \mathrm{~mm}$ diameter. The first one contained a small amount of the same nitroxide probe used as the label, in solution concentration in pure MBBA $\left(0.5 \times 10^{-6} \mathrm{~mol}\right.$ per gramme of MBBA). The concentration of the probe was kept very low, in order to have approximately the same intensity for the signal of the two samples and not to disturb the crystal. The nitroxide probe was dissolved in MBBA and the solution was directly degassed. The second sample contained silica grafted with chains labelled at their free end, with an excess of MBBA (about ten times the weight of the silica). First the grafted silica was dehydrated under vacuum for 4 hours and then the MBBA was added. At last all the samples were degassed under a dynamic vacuum of $10^{-6}$ torr for 24 hours with repeated fusion of the MBBA. The samples were then sealed under vacuum.

The EPR spectra were recorded on a Varian E-4 spectrometer operating at X-band at $9.15 \mathrm{GHz}$. The temperature was regulated by a Varian E-257 temperature controller. The magnetic field varied between $3200 \mathrm{G}$ and $3300 \mathrm{G}$. It is known [26] and we have verified that these values are sufficient for the liquid crystal to be aligned in average.

3.1.1 Order parameter of the spin label. - The rodlike molecules of the liquid crystal tend to align themselves in certain temperature ranges. The directors of the small clusters of aligned molecules are in general randomly oriented. In its nematic phase, it is easy however to align all the MBBA molecules of the sample in the direction of a strong external magnetic field [26]. The aligned molecules of liquid crystal are

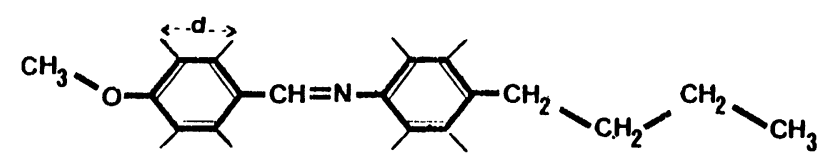

Fig. 2. - Chemical formula of MBBA. 
then rotating around their long molecular axis and influence the orientation of the spin probe, whose EPR signal reflects the local order. The orientational order of the liquid crystal is characterized by an order parameter $S$. In fact it is only the order parameter of the spin-probe which is experimentally measured with this technique. We use an order parameter $S$ which depends on the direction of the $2 \mathrm{p} \pi$ nitrogen orbital of the label relative to the axis of the liquid cristalline system and nearly of the magnetic field. Let us denote $\theta$ the angle between these two directions. Then the order parameter is given by

$$
S=\frac{1}{2}\left(3\left\langle\cos ^{2} \theta\right\rangle-1\right)
$$

where the bracket indicates a mean value. If the motion of the label is isotropic the order parameter is equal to zero. If the motion is anisotropic, and the axis of fast rotation is rather parallel in average to the direction of the $2 \mathrm{p} \pi$ nitrogen orbital $S$ will have a value intermediate between 1 and 0 .

To evaluate the order parameter from the EPR spectra we use the expression reported by Seelig $[27,28]$. Seelig calculates first the time averaged hyperfine tensor $A$ and $g$ tensor. These tensors are well known in the molecular frame $x, y, z$, where the $x$ axis is along the $\mathrm{N}-\mathrm{O}$ bond and the $z$-axis along the direction of the nitrogen orbital $2 \mathrm{p} \pi$ (perpendicular to the plane of the label). Let us denote $A_{x x}, A_{y y}, A_{z z}$ the components of the hyperfine tensor in the frame $x$, $y, z$. The essential step is to take into account the rotational symmetry of the motion around the director. It can then be shown that $[27,28]$

$$
S=\left(a_{\text {ani }}-a_{\text {iso }}\right) /\left(A_{z z}-a_{\text {iso }}\right)
$$

where $a_{\text {ani }}$ is the hyperfine splitting between two lines of the EPR spectrum and $a_{\mathrm{iso}}=\left(A_{z z}+2 A_{x x}\right) / 3$.

To account for the eventual difference between the polarities in the single crystal and in the liquid crystalline phase, Seelig [28] proposed to multiply the last expression of the order parameter by the fraction $a_{\text {iso }} / a_{\mathrm{N}}$.

$$
a_{\mathrm{N}}=\left(A_{\|}+2 A_{\perp}\right) / 3
$$

$A_{\|}$(resp. $A_{\perp}$ ) is the hyperfine splitting between two lines of the EPR spectrum when the common director of the molecules of the liquid crystal is parallel (resp. perpendicular) to the magnetic field direction.

\subsection{NUCLEAR MAGNETIC RESONANCE.}

3.2.1 Samples and apparatus. - For the NMR study, $75 \mathrm{mg}$ of MBBA were added to $8 \mathrm{mg}$ of dehydrated silica in a pyrex tube of $5 \mathrm{~mm}$ diameter. In order to have the same amount of liquid crystal for the same specific surface of silica another sample containing $150 \mathrm{mg}$ of MBBA and $20 \mathrm{mg}$ of grafted silica was prepared in a similar tube. Both mixtures were degassed and the tubes were sealed under vacuum.

The spectra were recorded on a wide line Varian 4210 A spectrometer at $25^{\circ} \mathrm{C}$, a temperature were the MBBA is in its nematic phase. We have chosen the same magnetic field of $3250 \mathrm{G}$ than for the EPR experiments, to make a comparison possible. The frequency of observation was $14.04 \mathrm{MHz}$.

3.2.2 Order parameter of the molecules of liquid crystal. - The NMR spectrum of two coupled protons at a distance $d$ from each other is composed of two lines. If $\theta$ is the angle between the external static magnetic field and the proton-proton axis, it is well known that the fine structure splitting between the doublet is given by (see for example (26))

$$
\Delta H=\frac{3}{2}\left(\hbar \gamma / d^{3}\right)\left(3\left\langle\cos ^{2} \theta\right\rangle-1\right)
$$

where $\gamma$ is the gyromagnetic factor.

The spectrum of the nematic liquid crystal MBBA consists of at least three lines. The central line arises from all the protons of the aliphatic tails or the polymers which undergo a fast isotropic motion. The four protons of each aromatic ring give rise to the two lines symmetric to the central one and characteristic of interacting protons. The order parameter $\sigma$ of the MBBA molecules is given by [26]

$$
\sigma=\left(d^{3} / 3 h \gamma\right) \Delta H
$$

where $d \simeq 2.45 \AA$ (Fig. 2).

\subsection{DifFerential SCANNING CALORIMETRY.}

3.3.1 Samples and apparatus. - Two sets of samples were prepared in capsules, covered, crimped shut, and placed in the DSC cell. The first one contains grafted silica and various quantities of MBBA. The second, ungrafted silica and various quantities of MBBA. For the first set, the grafted silica was dehydrated by direct pumping for 24 hours. The silica of the second set was dryed for $\mathbf{4 8}$ hours at more than $100^{\circ} \mathrm{C}$. To allow some comparison, a sample with pure MBBA was also prepared. The samples were used after waiting several days at about $10^{\circ} \mathrm{C}$, for a good silica-MBBA contact.

Transition temperatures were determined with a DuPont 1090 differential scanning calorimeter operating generally at a heating rate of $1^{\circ} \mathrm{C} / \mathrm{min}$ from about $-20^{\circ} \mathrm{C}$ at about $+50^{\circ} \mathrm{C}$. The measurements were performed every $5 \mathrm{~s}$ and recorded on a disk by a DuPont 1091 recorder. Before the measurements, a first run at a rate of $5^{\circ} \mathrm{C} / \mathrm{min}$ from $-20^{\circ} \mathrm{C}$ to $+40^{\circ} \mathrm{C}$ was made, to allow a good thermal contact.

\section{Results.}

4.1 EPR MEASUREMENTS. - For the samples with the spin probe in MBBA (control), the spectra consist of three lines characteristic of the nitroxide (Fig. 3b). 


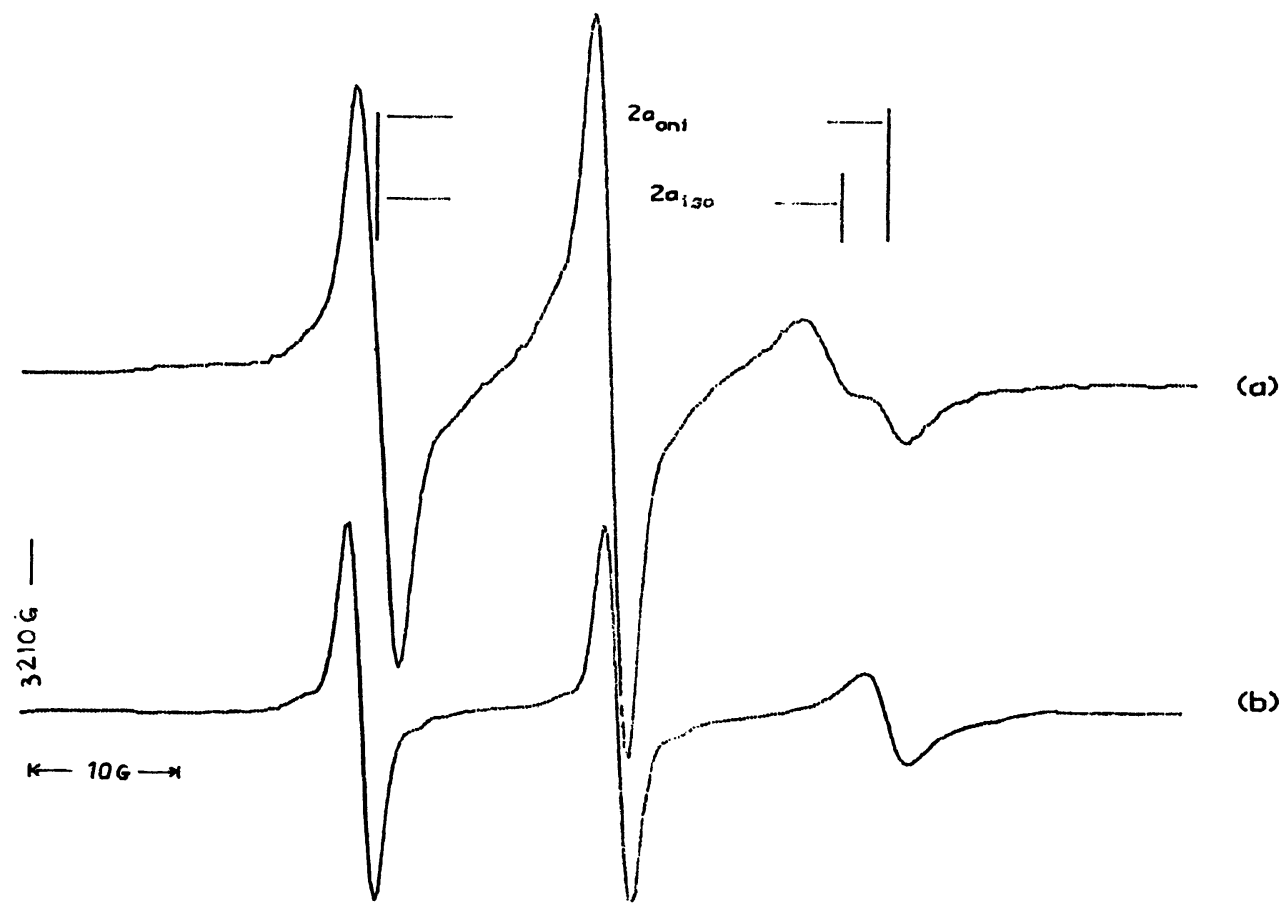

Fig. 3. - EPR spectra at $30^{\circ} \mathrm{C}$ of a) grafted labelled silica with MBBA, b) the probe in solution in MBBA (control).

The anisotropy of the motion is apparent from the spectra by an appreciable variation of the distance between the lines. This splitting decreases when the temperature increases until it reaches at $43{ }^{\circ} \mathrm{C}$ a value of $14.5 \mathrm{G} \mathrm{(Fig.} \mathrm{4),} \mathrm{which} \mathrm{is} \mathrm{similar} \mathrm{to} \mathrm{what} \mathrm{is} \mathrm{obtained}$ for an isotropic motion of the probe. The clarification point could be somewhat lower in this sample due to the presence of the probe. Indeed it has been checked by DSC.

When the liquid crystal is in contact with grafted silica two effects act simultaneously : the external magnetic field tends to align the molecules along its direction, and the solid surface induces another orientation. The EPR spectra are therefore much more complicated (Fig. 3a). They appear rather as a superposition of three spectra. One is due to the slow motion of the label adsorbed on the solid surface. Its shape has been described by Freed [29]. The outer extrema are well resolved and are distinct from the fast motion lines, whereas the central lines are all superposed. This slow motion spectrum disappears gradually when the temperature increases because the adsorption of the label becomes weaker. On the other hand the fast motion lines can be related to a distribution of the directors of the spin labels. We have interpreted it as a superposition of a spectrum characteristic of an isotropic fast motion of the label for which the splitting between the lines is $14.5 \mathrm{G}$, and another spectrum characteristic of an anisotropic fast motion. The splitting between the lines is greater and varies with temperature for this latter spectrum (Fig. 4). The central lines are all superposed, the high field lines

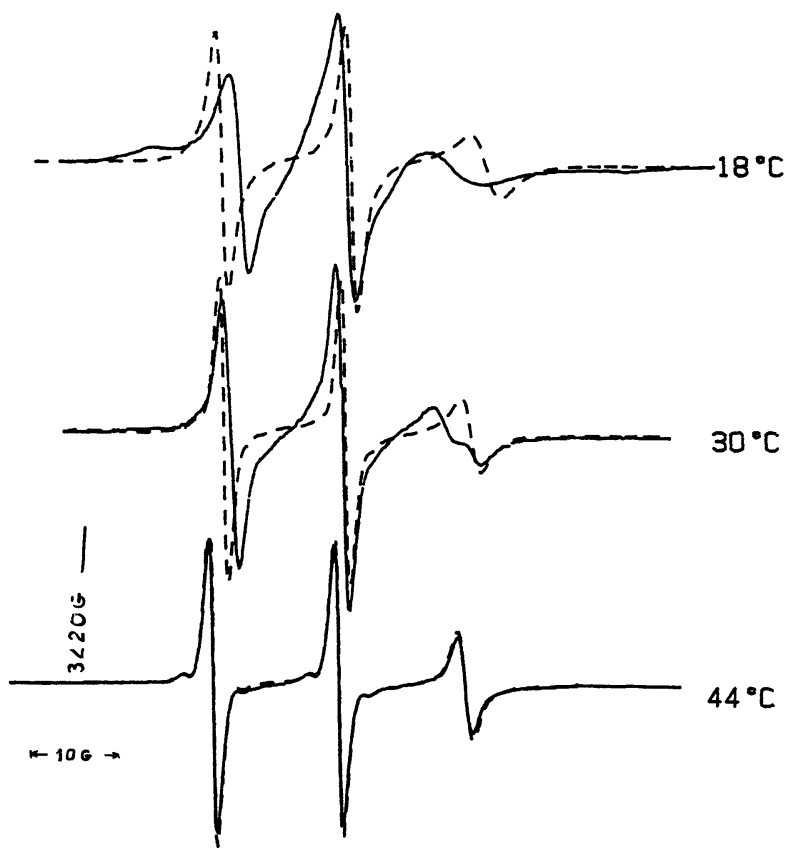

Fig. 4. - EPR spectra at different temperatures of (-) grafted labelled silica with MBBA and (-----) the probe in solution in MBBA.

are well resolved and the low field lines less resolved for the two fast motion spectra.

Usually the molecules of liquid crystal in the bulk nematic phase align their rotation axis roughly parallel to the magnetic field. The splitting between the spectral lines of the probe is then the component 
$a_{\mathrm{ani}}$ of the hyperfine tensor defined above. The splitting between the lines, $a_{\text {ani }}$ and $a_{\text {iso }}$ have been estimated by the distance of the shoulders on the experimental spectra as shown in figure 3 . The error on $a_{\text {ani }}$, for the sample with the spin probe in MBBA, is $3 \%$. For the sample with grafted silica and MBBA, the error on $a_{\text {ani }}$ and $a_{\text {iso }}$ is estimated to be $10 \%$.

The order parameter can therefore be calculated using the second expression of Seelig given [27, 28]. $A_{z z}=33.5 \mathrm{G}$ and $A_{x x}=5 \mathrm{G}$ were calculated from the rigid limit spectrum of the label in solution in benzene at $120 \mathrm{~K}$. We must remark that the geometry of the samples do not allow to point the molecules of the MBBA parallel and perpendicular to the magnetic field, and so to calculate $a_{\mathrm{N}}$. The error introduced by this difference of environments polarities is less than $6 \%[28]$. The experimental order parameters are given in figure 5. It can be noted that for temperatures below $20^{\circ} \mathrm{C}$ the motion of the label is again isotropic. This could be explained by the fact that the MBBA does not completely crystallize at low temperature as can be seen from the DSC curves (Fig. 7).

At a given temperature in the nematic region, the value of the order parameter for labelled grafted silica is clearly lower than for the probe in pure MBBA but still higher than in an isotropic liquid. Therefore

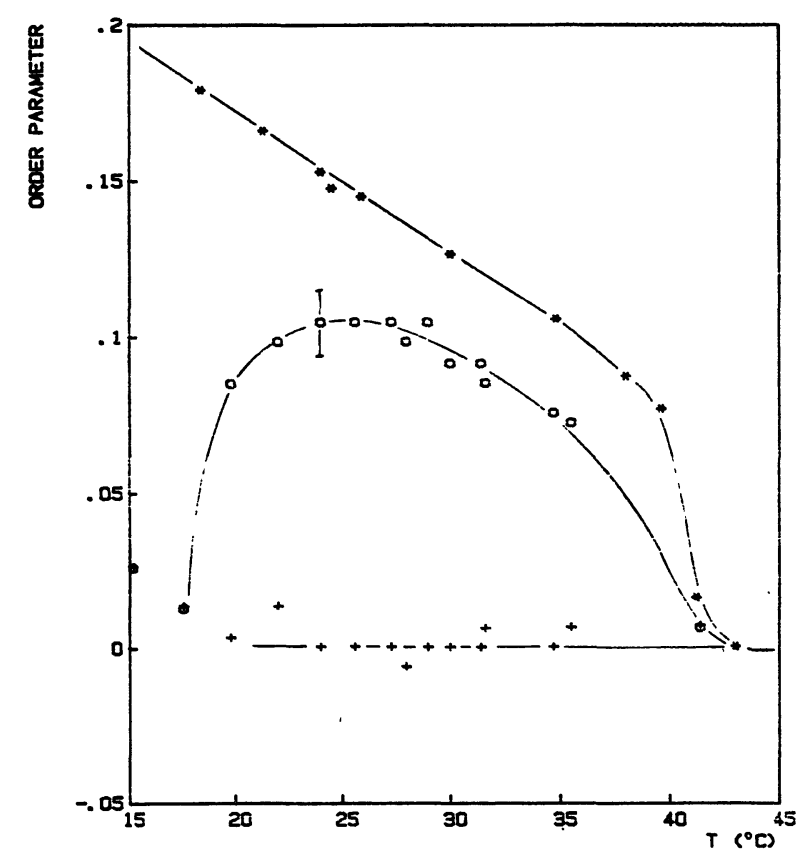

Fig. 5. - Order parameters for (*) the probe in solution in MBBA (control), (O) the oriented tails of the polymers of the grafted silica near MBBA, $(+)$ the disoriented tails of the polymers of the grafted silica near MBBA.

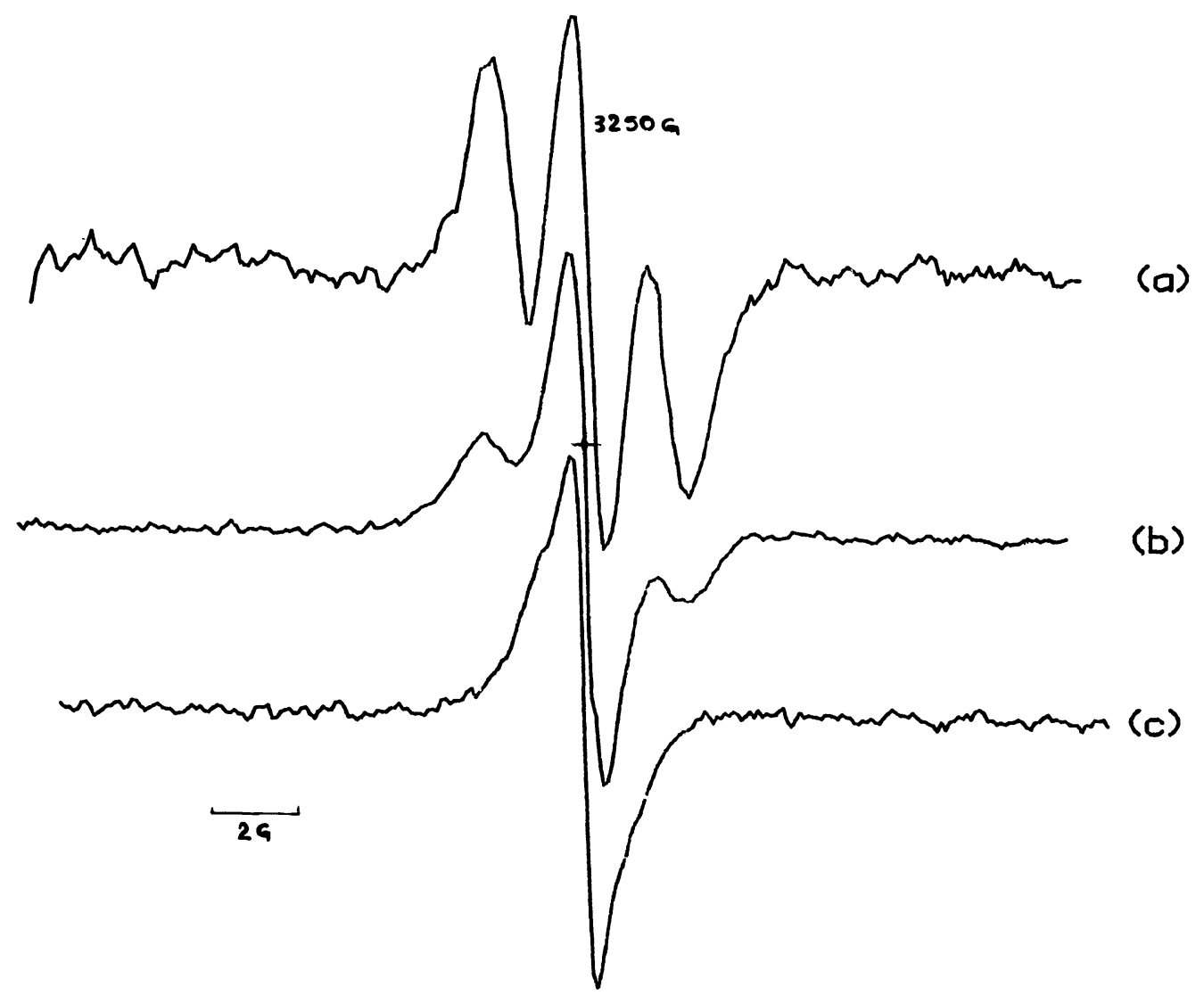

Fig. 6. - NMR spectra at room temperature of a) the pure MBBA (control); b) the MBBA in contact with a silica surface with grafted PEO; c) the MBBA in contact with a silica surface without PEO. 

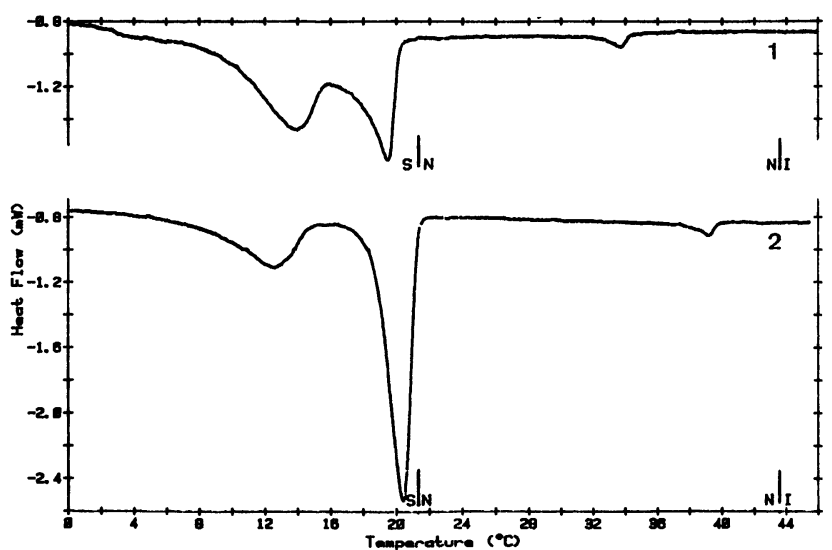

Fig. 7. - DSC trace of 1) ungrafted silica and MBBA; 2) grafted silica and MBBA. (1.3 g silica/7.5 g MBBA).

it appears that at the interface of grafted silica there exists an intermediate layer with polymers mixed with disoriented liquid crystal. The labels which are in this region undergo a fast isotropic motion. The labels which are directly adsorbed on the surface undergo a slow motion. Some tails protrude into the aligned liquid MBBA and the motion of the corresponding labels becomes anisotropic. Still the presence of the polymer tails perturb the nematic order; the chemical bonding of the label is another hindrance to its motion. As a result the labels are less oriented when fixed on a polymer tail than the probes are in the bulk. When the temperature increases both motions become more similar.

4.2 NMR MEASUREMENTS. - The MBBA rod contains four couple of protons of the aromatic rings which interact through the dipolar interaction. As the liquid crystal molecules are roughly aligned along the magnetic field, the resulting resonance line splits by an amount proportional to the order parameter. Here the probe is the liquid crystal itself and the measured order parameter is actually the order parameter of the liquid crystal. The other protons in the sample e.g. aliphatic tails of the MBBA or protons of the PEO undergo a fast isotropic motion and give rise to a simple central line in the spectrum.

The spectra are given in figure 6 for three samples at room temperature $\left(25^{\circ} \mathrm{C}\right)$. As expected $[1,26]$ the spectrum of pure MBBA (control) at this temperature consists of three lines : a central one arising from the tails of the molecules and the doublet characteristic of the local order. The greater the order, the greater is the splitting. When the motion is isotropic the splitting disappears. Here the lines are very well resolved indicating a good ordering of the liquid crystal in a magnetic field of $3250 \mathrm{G}$. The order parameter is about $S \simeq 0.58$.

For the liquid crystal near a silica surface with grafted polymer the central line is greater due to the protons of the polymer. The ordering of some of the molecules of liquid crystal is still detectable but the intensity of the doublet is relatively smaller and the order parameter is about $S \simeq 0.55$.

For the liquid crystal near a silica surface without grafted polymer, the doublet appears very weak in the bottom of the central line and the order parameter is probably less than $S=0.35$.

From these experiments the state of the liquid crystal in the bulk can be deduced. In all cases a fraction at least of the liquid crystal is aligned along the magnetic field. That fraction decreases when the MBBA is near a silica surface with grafted polymers and decreases still more near a silica surface without grafted polymers. The order parameter decreases following the same sequence.

4.3 DSC MEASUREMENTS. - The thermogram of pure MBBA (control) shows two peaks. A first one at $21^{\circ} \mathrm{C}$ corresponds to the solid-nematic transition. The second one at $44^{\circ} \mathrm{C}$ of weaker amplitude characterizes the nematic-isotropic transition.

For the MBBA interacting with a solid surface a third peak at about $14^{\circ} \mathrm{C}$ appears and the peak characteristic for the nematic-isotropic transition is shifted towards the low temperatures. If the quantity of MBBA is decreased, the intensity of the peak at $21^{\circ} \mathrm{C}$ corresponding to the solid-nematic transition in the bulk decreases. Therefore the peak at $14^{\circ} \mathrm{C}$ is characteristic of disordered liquid crystal near the solid surface (Fig. 7).

Comparing the effect of silica with and without grafted polymers it appears that the peak at $14^{\circ} \mathrm{C}$ is more important and the nematic-isotropic transition occurs at lower temperature for the silica without grafted polymers (Fig. 8). The two low temperatures peaks have been decomposed for the samples with $1.3 \mathrm{~g}$ of silica for $7.5 \mathrm{~g}$ of MBBA. The $14^{\circ} \mathrm{C}$ peak represents $40 \%$ of the total area for silica without polymer. It represents only $30 \%$ of the total area for grafted silica.

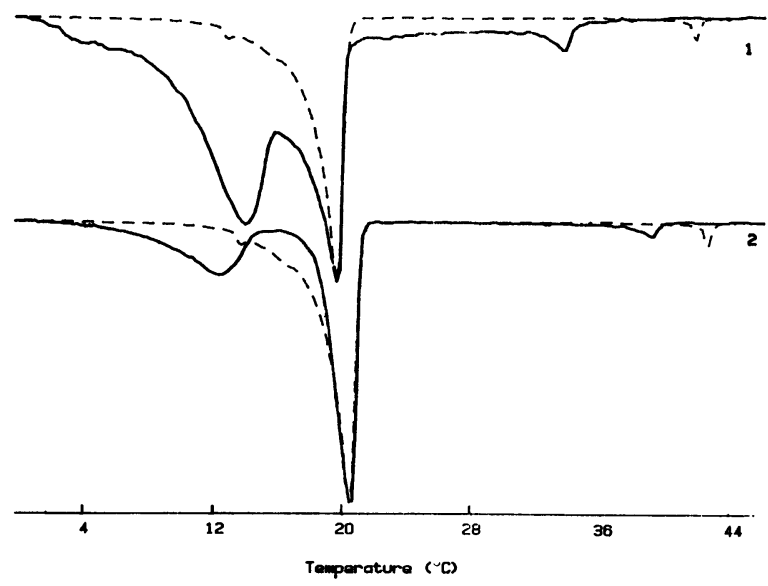

Fig. 8. - Decomposition of the DSC trace of 1) ungrafted silica and MBBA and 2) grafted silica and MBBA. 
The results confirm the precedent conclusions drawn from the NMR measurements. There exists always an isotropic disordered phase near the silica surface. However, when polymers are grafted on the surface the perturbations are weaker. In the bulk the nematic order is higher and the temperature range of existence of a nematic phase is larger.

\section{Discussion.}

5.1 EXPECTED PHENOMENA. - For the local resonance studies (EPR, NMR) a very large surface was necessary because the sensitivity of the technics are relatively low. That is why a pyrogenic Aerosil silica of $310 \mathrm{~m}^{2} / \mathrm{g}$ was used. Even in contact with such a surface, a fraction at least of the liquid crystal is ordered in a nematic phase. However the specific properties of this surface must be recalled in order to understand the results. It is well known that the orientation of the liquid crystal depends strongly on the state of the solid surface. Two types of parameters are important in this respect : the geometry of the surface [2] and the physico-chemical couplings $[5,6]$.

The Aerosil silica is non-porous. But its preparation proceeds in different stages. First colloidal particles are formed and they grow spontaneously to recognizable size (in our case about $10 \mathrm{~nm}$ diameter). These particles as oligomers aggregate then, until they form chains of particles, polymers, and gel networks of silica [30]. The surface is therefore highly irregular, and though it is not porous, at the scale of the MBBA molecules, no preferential orientation of the liquid crystal can be expected.

Concerning the physico-chemical coupling, the polar $\mathrm{Si}-\mathrm{OH}$ groups are the sites at which adsorption occurs [30]. When the silica surface is covered with poly(ethylene oxide) chains these interactions are of course modified. Silica itself is hydrophilic. We have chosen PEO as the polymer, because it is miscible with water in all proportions at room temperature. With the grafting ratio used, the chains interact with each other and adopt a configuration with loops, trains and tails [23-25, 31, 32]. The bond dipole moments are somewhat different for the surface and the chains [33]. However compared to the large difference in chemical nature between the polymer and the solvent (as reflected in solubility parameters, for instance) [19] the physico-chemical interactions are not so much modified.

Therefore at the interface between silica and the liquid crystal MBBA, there will not be strong orienting interactions. With grafted polymers, the surface will only become slightly more organophilic. In each case no definite anchoring orientation is expected.

5.2 ObSERVATIONS. - All experimental techniques used show evidence for a disordered layer at the interface between the silica and the MBBA in the interesting temperature range. However this intermediate layer is larger with silica than with grafted silica :

Disorder near silica without PEO >

$>$ silica with grafted PEO.

An explanation for this behaviour could be the following. At the surface, the number of nearest neighbours of a MBBA molecule is smaller than in the bulk. As a consequence, the surface may start to disorder as the transition is approached although the bulk is still in its ordered state. Thus a layer of the disordered phase intervenes between the surface and the bulk, and the material undergoes a surface induced disorder transition. Such a transition occurs if the microscopic interaction parameters are comparable or weaker than those in the bulk [34] as it is the case for the silica surface.

Clearly the polymer displays a very important influence on the properties of the interface. As can be seen qualitatively from the EPR experiments, the polymers and in particular the tails fill the gap and holes into the liquid crystal lattice. Moreover the loops, and the polymer layer formed, smooth out the surface. As a result, a certain decoupling of the solid silica surface from the liquid crystal is achieved, and the order of the MBBA molecules is enhanced.

Quantitatively from the DSC data at $14^{\circ} \mathrm{C}$, the thickness of the disorder layer can be estimated. Considering the occupied surface of a MBBA molecule to be $50 \AA^{2}$, this thickness is about $150 \AA$ with grafted polymers and $200 \AA$ for the pure silica. The radius of gyration of the polymer is about $15 \AA$. From earlier calculations $[1,2]$, it is expected that the order of magnitude of the distance on which the liquid crystal should be perturbed by an irregular surface is precisely the order of the irregularities (i.e. here the size of the silica particles $100 \AA$ ). Our results are consistent with these predictions. The order of the nematic liquid crystal decreases slowly when approching the surface but still influences the orientation of the polymer tails (Fig. 9).

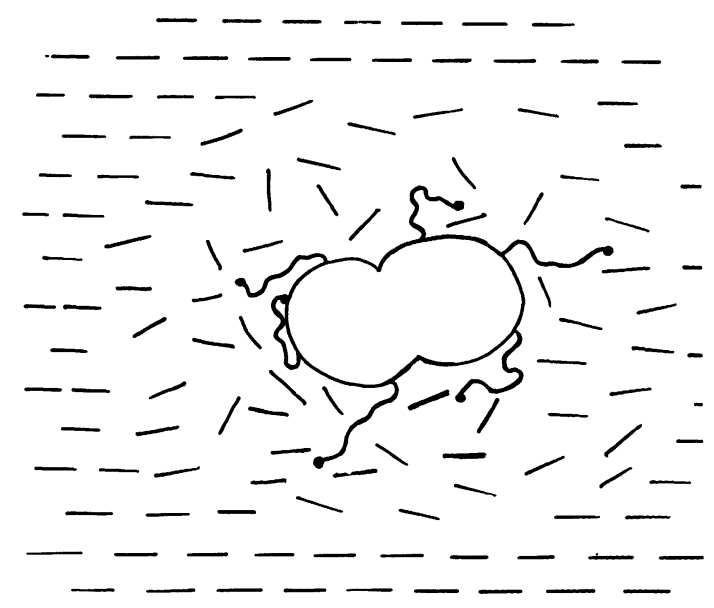

Fig. 9. - Proposed model of the nematic liquid crystal near a wall with polymers. 
In solution Kronberg et al. [19] have shown that polymers destroy the nematic order. Here with grafted polymer onto a solid surface we have shown a completely different behaviour. In fact all the components of the new system must be taken into account in order to explain the results $[25,31]$. There are three components which interact with each other : the solid, the polymer and the solvent. In our case the polymer is adsorbed on the wall ; both the wall and the polymer influence the nematic order. This leads to experimental effects which are more complicated than the predictions of the simplest models would give.

\section{Conclusion.}

The ordering properties of a nematic liquid crystal has been studied in the vicinity of a solid silica surface with grafted polymers, using the EPR, NMR and DSC techniques. The results from all techniques suggest that there exists an intermediate interfacial layer with polymers mixed with liquid crystal, where the ordering is weak. However when compared with the effect of pure silica, it has been shown that the grafting of polymers greatly improves the decoupling of the bulk nematic liquid crystal from the surface. This effect can be interesting for our understanding of the behaviour of liquid crystals near walls and for practical applications.

\section{Acknowledgments.}

We thank F. Brochard and P. G. de Gennes for their encouragements and C. Noël for her helpful advice.

References

[1] De Gennes, P. G., The Physics of liquid crystals (Oxford University Press, London), 1974.

[2] Berreman, D. W., Phys. Rev. Lett. 28 (26) (1972) 1683.

[3] Cheng, J., Boyd, G. D., Appl. Phys. Lett. 35 (6) (1979) 444.

[4] Urbach, W., Borx, M., Guyon, E., Appl. Phys. Lett. 25 (9) (1974) 479.

[5] Proust, J. E., Ter-Minassian-Saraga, L., Guyon, E., Solid State Commun. 11 (1972) 1227.

[6] Kahn, F. J., Taylor, G. N., Schonhorn, H., Proc. IEEE 61 (7) (1973) 823.

[7] Haller, I., Appl. Phys. Lett. 24 (8) (1974) 349.

[8] PORTe, G., J. Physique Colloq. 37 (1976) 1245.

[9] Naemura, S., J. Physique Colloq. 40 (1979) C3-514.

[10] Miyano, K., J. Chem. Phys. 71 (10) (1979) 4108.

[11] Hiltrop, K., StegemeYer, H., Ber. Bunsenges Phys. Chem. 85 (1981) 582.

[12] Proust, J. E., Ter Minassian-Saraga, L., J. Physique Colloq. 36 (1975) C1-77.

[13] Rivière, D., LÉvy, Y., GuYon, E., J. Physique Lett. 40 (1979) L-215.

[14] Yang, K. H., J. Appl. Phys. 53 (10) (1982) 6742.

[15] Yang, K. H., Rosenblatt, C., Appl. Phys. Lett. 43 (1) (1983) 62.

[16] Mada, H., Kobayashi, S., Appl. Phys. Lett. 35 (1) (1979) 4.

[17] Salamon, Z., Skibinski, A., Mol. Cryst. Liq. Cryst. 90 (1983) 205.

[18] Van Sprang, H. A., J. Physique 44 (1983) 421.

[19] Kronberg, B., Bassignana, J., Patterson, D., J. Phys. Chem. 82 (1978) 1714.

[20] Dubault, A., Casagrande, C., Veyssie, M., Mol. Cryst. Liq. Cryst. Lett. 41 (1978) 239.
[21] Dowell, F., Martire, D. E., J. Chem. Phys. 69 (6) (1978) 2322.

[22] Brochard, F., C.R. Hebd. Séan. Acad. Sci. 289 (1979) 229 ; C.R. Hebd. Séan. Acad. Sci. 290 (1980) 485; Communication to the 27th International Symposium on Macromolecules. Strasbourg 6-9 July 1981, p. 768.

[23] Hommel, H., Legrand, A. P., Balard, H., PaPiRer, E., Polymer 24 (1983) 959.

[24] Hommel, H., Legrand, A. P., Tougne, P., Balard, H., PAPIRER, E., Macromolecules 17 (1984) 1578.

[25] Hommel, H., Legrand, A. P., Balard, H., PaPirer, E., Polymer 25 (1984) 1297.

[26] Papon, P., Le Pesant, J. P., Chem. Phys. Lett. 12 (1971) 331.

[27] Seelig, J., J. Am. Chem. Soc. 92 (13) (1970) 3881.

[28] SeELig, J., in Spin Labelling Theory and Applications, Ed. L. J. Berliner (Academic Press, New York) 1976.

[29] Freed, J. H., in Spin Labelling Theory and Applications, Ed. L. J. Berliner (Academic Press, New York) 1976.

[30] ILER, J. K., The Chemistry of Silica (John Wiley \& Sons Interscience New York) 1979.

[31] Howard, G. J., McConnell, P., J. Phys. Chem. 71 (9) (1967) 2974.

[32] FaCChINI, L., Legrand, A. P., Macromolecules 17 (1984) 2405.

[33] FloRY, P. J., Statistical Mechanics of chain molecules (John Wiley \& Sons Interscience, New York) 1969.

[34] Lipowsky, R., J. Appl. Phys. 55 (6) (1984) 2485. 